\title{
SERIOUS CONGENITAL HEART DISEASE IN INFANCY
}

\author{
BY \\ ERIC N. COLEMAN \\ From the Department of Cardiology, Royal Hospital for Sick Children, and the University Department of Child Health, \\ Glasgow
}

Received February 10, 1964

During a three-year period ending in 1962, 166 children in the first year of life were admitted to the Royal Hospital for Sick Children in Glasgow because of symptoms directly attributable to serious congenital heart disease. Of these, 96 died before their first birthday, accounting for onefifth of all deaths in the first year of life and for every seventh death among all admissions to the hospital. These figures, which take no account of the larger number of infants admitted for other reasons and found to have congenital heart disease, contrast with a total of 6 admitted during the first triennium of the present Royal Hospital for Sick Children which ended in 1917. This increase in hospital incidence with its high mortality also contrasts sharply with the general decline in infant disease and mortality of the intervening 45 years, at the beginning of which the impact of congenital heart disease on an infant population grievously afflicted by infection was negligible.

The objects of this paper are to classify the lesions, to describe their immediate prognosis, and to consider the fitness of these infants for surgical treatment.

\section{Clinical Material}

The clinical material consisted of all infants (children in the first year of life) admitted to the medical wards from the out-patient clinics or directly from home or maternity hospitals because of unequivocal symptoms of congenital heart disease during the three-year period October 1, 1959 to September 30, 1962. The survey ended at the first birthday or at death if this occurred during the first year of life.

Presenting symptoms regarded as significant were breathlessness at rest or on exertion as reflected in slow feeding or refusal of feeds, central cyanosis at rest or on exertion as when crying, and failure to thrive without other cause. The diagnosis was made in $\mathbf{4 5}$ patients by clinical, radiological, and electrocardiographic examination and in the remaining 121 this was supplemented by information obtained from cardiac catheterization (52), thoracotomy (38), or necropsy (80).

In order to relate deaths to those who survived without surgical treatment, the term net mortality has been used, since operation was only attempted as a possible life-saving measure, and the inclusion of those who survived because of it would underrate the gravity of the prognosis of an untreated group.

Patients suffering from congestive heart failure were propped up, nursed in oxygen, and digitalized in 24-36 hours; digoxin was continued as maintenance therapy with low sodium milk ("Edosol") and thiazide diuretics as occasional adjuvants.

\section{RESULTS}

There were 166 patients who came under observation of whom $96(58 \%)$ died. Since 15 of the survivors received surgical treatment the net mortality was 64 per cent. There was a male preponderance of $93(56 \%)$ to $73(44 \%)$, but the gross mortality rates by sex were almost identical; 58 per cent of the males died ( 54 deaths) and 57 per cent of the females ( 42 deaths). 
TABLE I

Prognosis Related to Cyanosis and Congestive Heart Failure

\begin{tabular}{c|c|c|c}
\hline \multicolumn{2}{c|}{ Category } & Net mortality (\%) & $\begin{array}{c}\text { Chance of survival to first } \\
\text { birthday (approx.) }\end{array}$ \\
\cline { 1 - 2 } Cyanosis (+ or - ) & Heart failure (+ or - ) & & $3: 1$ \\
\cline { 1 - 2 } & - & 24 & 1 \\
+ & $\bar{t}$ & 74 & $0.3: 1$ \\
+ & + & 90 & $0 \cdot 1: 1$ \\
\hline
\end{tabular}

Cyanosis. Clinical cyanosis was present in 83 patients and absent in 83 . The epithet is used because 6 patients did not appear cyanosed, though their cardiac malformations were such as to cause systemic arterial hypoxia: 2 with single ventricle, 1 with total anomalous pulmonary venous drainage, 1 with persistent truncus arteriosus, 1 with right ventricle with double vascular outlet, and 1 with Taussig-Bing complex. There were 39 deaths in the acyanotic group and 57 in the cyanotic group, representing net mortality rates of 55 and 76 per cent respectively.

Congestive Heart Failure. 96 infants $(58 \%$ ) were already in congestive heart failure when referred, and 75 died, a net mortality of 83 per cent. In contrast, the net mortality was only 34 per cent among the 70 infants who did not present in heart failure. Of those presenting in failure, 49 were cyanosed and 47 were not; the net mortality of the cyanotic group was 90 per cent as against 76 per cent of the acyanotic group.

Division of the entire sample into 4 groups according to the presence or absence of cyanosis and of congestive heart failure at the time of referral (Table I) revealed that for the cyanotic infant the chance of survival to the first birthday was 3 times less than for the acyanotic, and that the presence of congestive heart failure reduced tenfold the chance of survival of both the cyanotic and acyanotic. The acyanotic infant without congestive failure had 3 chances in 4 of surviving, the cyanotic with congestive failure only 1 chance in 10 .

Age and Urgency. $138(83 \%)$ were referred during the first six months of life and $105(63 \%)$ of them before the age of 3 months. Of those who presented in congestive failure $90(94 \%)$ did so before the age of 6 months and $80(83 \%)$ before the age of 3 months. Age at the time of death followed a similar pattern, and $73(76 \%)$ occurred in the first 6 months of life. Further emphasis on urgency is given by the observation that $37(39 \%)$ of the deaths took place within one week of referral to hospital.

The Common Malformations. A most serious difficulty that besets a classification of the forms of congenital heart disease that are important during very early life when multiple lesions are frequent is the avoidance of diagnostic over-simplification. On the other hand too much detail somewhat defeats the object, and in cases with several malformations only the one that from the evidence available is regarded as having had the most profound effect on circulatory hæmodynamics is indicated. Shunts through defects essential to life in, for example, complete transposition of the great arteries, tricuspid atresia, and total anomalous pulmonary venous drainage are regarded as inseparable from the primary lesion.

On this basis the 9 most frequent lesions, which were diagnosed in 138 patients $(83 \%)$, are set out in Table II. Ventricular septal defect, the principal lesion of 42 patients $(25 \%)$, was first in frequency followed by complete transposition of the great arteries and by pre-ductal coarctation of the aorta with its compensating ductus arteriosus. One of these three was the principal lesion of 84 patients $(50 \%)$. In the absence of a generally accepted classification, the term pre-ductal coarctation is here used to signify all grades of narrowing of the aortic arch. Other common lesions (54 patients) in order of decreasing frequency were persistent ductus arteriosus, endocardial fibro-elastosis, tetralogy of Fallot, persistent truncus arteriosus, tricuspid atresia, and total anomalous pulmonary venous drainage. The remaining 28 patients presented single or multiple lesions of lesser frequency 
TABLE II

Principal Lesions in $138(83 \%)$ of 166 Patients

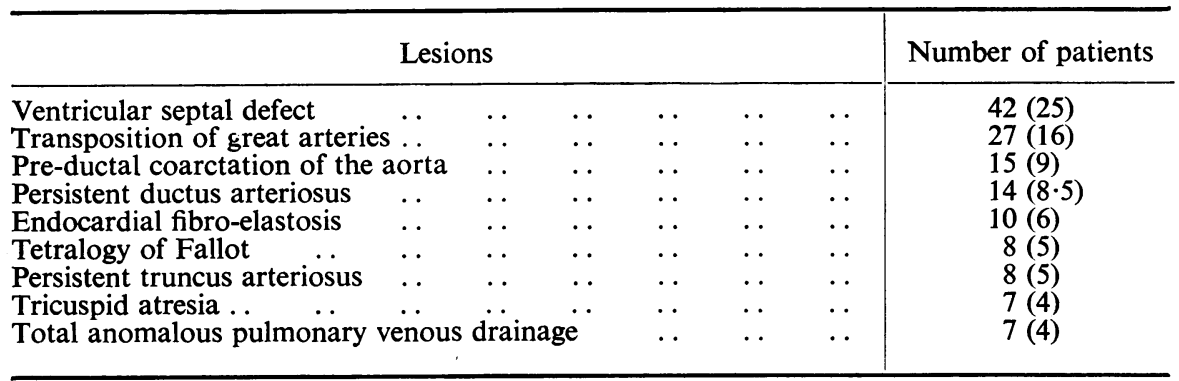

Percentages are given in parenthesis.

TABLE III

Principal Lesions in $80(83 \%)$ of 96 Patients who Died

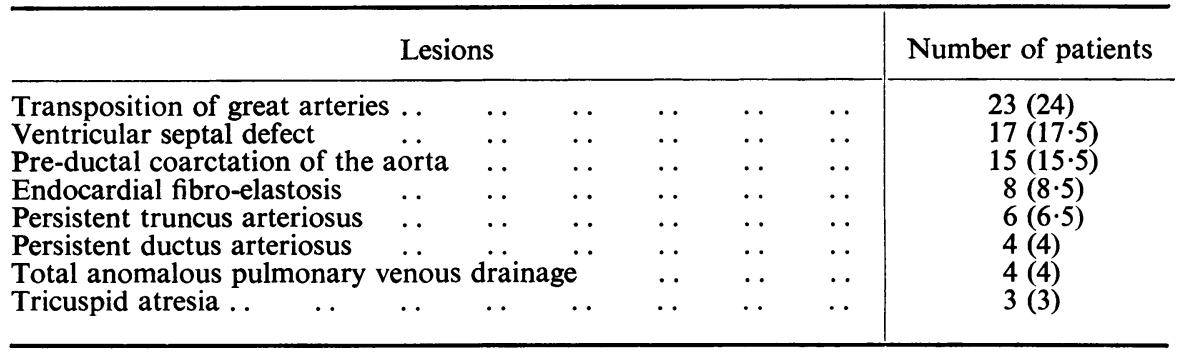

Percentages are given in parenthesis.

including single ventricle, cor biloculare, right ventricle with double vascular outlet, and aortic stenosis.

Fatal Malformations. The 8 principal lesions causing death in $80(83 \%)$ of 96 patients are given in Table III. The same lesions, namely, complete transposition of the great arteries, ventricular septal defect, and coarctation of the aorta, headed this list and accounted for the death of 55 patients $(57 \%)$. As a cause of death complete transposition of the great arteries ranked higher than ventricular septal defect; the former malformation caused 23 deaths $(24 \%)$, the latter $17(17 \cdot 5 \%)$. Other fatal lesions in order of decreasing frequency were endocardial fibro-elastosis, persistent truncus arteriosus, persistent ductus arteriosus, total anomalous pulmonary venous drainage, and tricuspid atresia. The remaining 17 patients presented a variety of lesions none of which occurred in more than two. The tetralogy of Fallot did not cause a single death in this age-group.

Further information was sought with regard to those patients who died of the three most common malformations.

Transposition of the Great Arteries. The mean age at death of the 23 patients was 14 weeks (range, 17 days to 11 months): 7 died within one week of referral; 6 of these and 11 others were already in congestive heart failure when referred. Of the 7 patients who died soon after referral one had in addition a hypoplastic pulmonary valve and artery; another had pyogenic meningitis. Of the 16 who died later, one had tricuspid atresia and pyogenic meningitis, one had renal vein thrombosis, and a third had pyogenic meningitis without any additional cardiac lesion. One of the 4 survivors had tricuspid atresia, one pulmonary stenosis, and two had grossly dilated pulmonary arteries demonstrated by angiocardiography.

Ventricular Septal Defect. The mean age at death of the 17 patients was 17 weeks (range 5 weeks to 11 months). None died of an uncomplicated ventricular septal defect. Three died 
within one week of referral in congestive heart failure, and in each instance two of three complications were present, namely pulmonary hypertension, additional cardiac lesions, or bronchopneumonia. Of 4 who died soon after an emergency operation, all had pulmonary hypertension, 3 had presented in congestive failure, and 2 had additional cardiac lesions. Of the remaining 10, 6 presented in congestive failure, 7 had pulmonary hypertension, 4 had additional cardiac lesions, and in 3, death was precipitated by bronchopneumonia. The additional cardiac lesions were atrial septal defect, tricuspid incompetence, pulmonary valve stenosis, pulmonary infundibular stenosis, left atrial fibro-elastosis, and overriding aorta.

Seven patients who presented in congestive heart failure survived; 3 with pulmonary hypertension were treated by pulmonary artery banding; one had an accompanying patent ductus arteriosus ligated, and in the remaining 3 there was evidence of pulmonary infundibular stenosis.

Coarctation of the Aorta. The mean age at death of the 15 patients with pre-ductal coarctation of the aorta was 7 weeks (range, 5 days to 18 weeks). All but one were in congestive heart failure when referred: 8 died within a week, of whom 5 had cardiac lesions additional to the primary malformation with its compensating ductus arteriosus; 5 died soon after an emergency operation, and 3 of these had additional lesions; one of the 2 remaining patients had an additional cardiac lesion. The additional lesions encountered were ventricular septal defect, atrial septal defect, and aortic stenosis, alone or in combination.

\section{Discussion}

The increased incidence of serious congenital cardiovascular disease during the last 45 years amongst infants seen at the Royal Hospital for Sick Children in Glasgow does not necessarily reflect an increased incidence within the population at large. The issue has become inextricably confused by important factors such as the virtual elimination of some fatal infections, the formation of a universal health service, and a greater awareness of the disease stimulated by the increasing proficiency of heart surgery. It is not even possible to state that the experience of one hospital can be taken to reflect the national incidence, a subject that will receive comment with regard to individual lesions. If it is assumed, however, that the Royal Hospital for Sick Children, Glasgow, caters for the infants of a population of 2 millions, that this represents approximately 4 per cent of the population, and that regional variations in the over-all incidence are not large, it can be estimated that each year at least 1400 infants with symptoms caused by congenital heart disease present at pædiatric departments in the United Kingdom, and that 800 may die during the first year of life. This estimate takes no account of the considerable number who die in maternity hospitals.

A numerical assessment has been made of the sinister effect on prognosis of the early appearance of congestive heart failure and of the more dismal prognosis conveyed by the cyanotic types of congenital heart disease. In practice, the presence or absence of these two features appears to give an indication of the prognostic pattern when the precise diagnosis may still be in doubt. One implication is clear; all measures necessary to establish an accurate diagnosis should be put in hand as a matter of urgency when heart failure appears during the first year of life, and particularly when this accompanies cyanotic congenital heart disease.

The serious and fatal malformations of Glasgow infants are substantially the same as those encountered by Keith (1956) as causing heart failure in his series of infants in Toronto. Complete transposition of the great arteries, ventricular septal defect, and coarctation of the aorta are the leading three lesions in both series, although their relative incidence is different. His inclusion of aortic atresia as fourth in frequency is, however, at variance with our experience in Glasgow both at the Royal Hospital for Sick Children, where in the 3 years under review no patient had this lesion, and at the associated pædiatric department of the Royal Maternity Hospital, where it is regarded as a very uncommon lesion.

Although Fallot's tetralogy accounted for 5 per cent of referrals, none presented in heart failure and none died in the first year of life. This with Keith's experience (1956) that rather less than 1 per 
cent of his cases of heart failure in the first year of life were attributable to Fallot's tetralogy contrasts strikingly with Bonham Carter's series (1955) from London in which Fallot's tetralogy when taken together with pulmonary stenosis had the third place among lesions responsible for fatal heart failure at this age. Even the addition of pulmonary stenosis does not raise this diagnostic grouping to a position of prominence in the Toronto series or in the present Glasgow series in which pulmonary stenosis was only once recorded as responsible for heart failure and death. Bonham Carter's figures (1955) also indicated that atrial septal defect was second in frequency as a cause of fatal heart failure in the first year of life. Far from being common, this defect was not once encountered as an isolated or principal lesion in the present series; neither does it appear in Keith's classification. In addition to the rare genetic transmission of atrial septal defect as an autosomal dominant characteristic (Zetterqvist, 1960; Holt and Oram, 1960), a recessive mode of inheritance has been claimed (Carleton, Abelmann, and Hancock, 1958) that might explain the low incidence of atrial septal defect among infants in Glasgow and Toronto as compared with its high incidence in London. The lower incidence in the Toronto series might be partly attributable to the genetic constitution conveyed to Ontario by its many early immigrants of Celtic stock, on the assumption that the recessive gene determining the occurrence of atrial septal defect may have a higher frequency amongst individuals of Anglo-Saxon descent. At the very least these different rates of incidence of atrial septal defect, Fallot's tetralogy, and aortic stenosis imply that statistics of congenital cardiovascular malformations are likely to be of dubious relevance for populations outside the immediate range of a survey.

Successful surgical treatment must depend upon its technical excellence and also upon the fitness of the infant for operation. Major complications must influence the outcome of treatment for the primary lesion. The presence of a second cardiovascular malformation was found to be an important problem by Ochsner et al. (1962) in an analysis of their "aggressive approach" to surgical treatment in infancy, and Gerbode and Cooley (1963) have added a word of caution to prevent undue optimism in connexion with the repair of intracardiac defects. These observations led to a consideration of the adverse circumstances in the natural history likely to affect the outcome of surgical treatment of the three foremost fatal lesions in Glasgow.

Pre-ductal coarctation of the aorta conferred a disastrous prognosis. Congestive heart failure appeared early and with little warning. As a rule only a brief interval elapsed between its onset and death; half of the patients were dead within a week of referral and most of these within the first 48 hours. Deterioration so rapid, so advanced by the time of referral, and so little influenced by medical treatment, allows little hope for the success of surgical treatment. In the majority moreover, the issue would have been prejudiced by the presence of additional cardiac lesions. Surgical treatment of 8 patients who had survived beyond a few days failed to prolong life. It would not be unrealistic to suggest that attempted surgical treatment of most other patients would merely have shifted the locus of death from ward to operating theatre. Nevertheless from the results of this study it would be reasonable to conclude that any infant who is believed to have coarctation of the aorta and who presents in congestive heart failure should be subjected to immediate thoracotomy after rapid digitalization; the delay and added danger inherent in cardiac catheterization and angiography should be avoided unless the diagnosis is seriously in doubt. The certainty is that a few patients whose narrow aortic segment is short and who do not have additional lesions will be saved; the mortality rate could scarcely be increased. There were in addition three instances of post-ductal coarctation of the aorta, two of whom had accompanying patency of the ductus arteriosus. These have not been included in Table II because of their low frequency. The one with no ductus presented with heart failure and survived the first year of life. The others did not have heart failure and were operated upon successfully.

Infants who died of complete transposition of the great arteries survived on average twice as long as those with pre-ductal coarctation of the aorta, but even so one-third were dead within a week of referral. Again the high frequency of congestive heart failure, present in three-quarters at the time of referral, must be taken as a sign of ill omen as regards prospects for successful surgical 
treatment. Nevertheless the infrequency of additional lesions, present in less than 10 per cent, was encouraging. The insidious onset of acute pyogenic meningitis in 3 patients was disquieting because in 2 the diagnosis was not made before death; it is possible that this complication is analogous to the cerebral abscess of Fallot's tetralogy and related to the direct communication between systemic veins and systemic arteries common to both conditions. The advanced state of deterioration of most patients at the time of referral as judged from the early occurrence of death and the presence of congestive failure gives the impression that surgical treatment would probably have been unavailing in 30 per cent and extremely hazardous in a further 45 per cent. Furthermore, all that can be offered is palliative treatment which, even in its most refined form (Senning, 1959), entails a hazardous operation and does not achieve redirection of the ventricular outflow tracts. Before the present study began the disappointing results of surgical treatment had led to its virtual abandonment, but here again the prognosis is so grave that there seems at worst little to be lost by its reintroduction. At best, there is a slender chance of preserving life long enough to allow for the development and subsequent application of more satisfactory surgical techniques.

Where ventricular septal defect was the principal lesion the prognosis was distinctly better. Though almost three-quarters of those who died presented in congestive heart failure, rather less than 20 per cent of the deaths occurred in the first week after referral. Unfavourable features were a high incidence of pulmonary hypertension and of additional cardiovascular lesions. Bronchopneumonia contributed to the death of a few patients and was responsible for congestive heart failure in two who died within a few days of referral. Infants with none of these complications seem almost certain to survive the first year of life. Congestive heart failure was not a feature of an uncomplicated ventricular septal defect, and in the ordinary course of events must be regarded as a sign of grave prognostic significance except perhaps where pulmonary hypertension is modified by pulmonary infundibular stenosis. In general, deterioration was not so rapid as with the two malformations already discussed and more might have been saved by prompt surgical treatment. Spontaneous closure of a defect that had caused heart failure was not encountered, and in any case this event has been regarded by Wade and Wright (1963) as unpredictable. The practice in this hospital is to expedite the investigation of all infants with ventricular septal defects and congestive heart failure in order to assess the degree of pulmonary hypertension in preparation for palliative banding of the pulmonary artery (Muller and Dammann, 1952), and to search for additional lesions that may prove to be operable. Digitalization would be mandatory and antibacterial therapy in full therapeutic dosage occasionally so. Because of the risk and possible effect of bacterial pneumonia there is much to be said for prophylactic antibacterial therapy in all those with symptoms, whether or not congestive heart failure has made its appearance.

\section{SUMmARY AND CONCLUSIONS}

Of 166 children in the first year of life recently referred to the Royal Hospital for Sick Children, Glasgow, because of symptoms attributable to congenital heart disease, 58 per cent died before their first birthday. Ventricular septal defect, complete transposition of the great arteries, and pre-ductal coarctation of the aorta were the most frequent lesions and, with transposition of the great arteries in the lead, were also the most frequent causes of death. Most were referred and died during the first six months of life. The early appearance of congestive heart failure reduced the chance of survival tenfold.

The geographical distribution of lesions shows wide variation, perhaps due to genetic factors. Glasgow and Toronto infants appear to have a pattern of incidence unlike that of London infants.

The serious prognosis of pre-ductal coarctation of the aorta and of complete transposition of the great arteries justifies an attempt at surgical treatment, but a high operative mortality is inevitable because of complications of the primary lesion.

Pulmonary hypertension and additional cardiac malformations complicating ventricular septal defects may precipitate congestive heart failure, and in this event palliative surgical treatment is indicated. 
In order to reduce mortality from severe congenital heart disease in the first year of life, better methods must be found to reconstruct the hypoplastic aortic arch, to redirect ventricular outflow to transposed great vessels, and to relieve pulmonary hypertension associated with defects in the ventricular septum.

I am indebted to physicians at the Royal Hospital for Sick Children for access to patients under their clinical care, to Professor J. H. Hutchison for criticism of the manuscript, to Mr. R. S. Barclay and his colleagues for reports of their operative findings, to Dr. A. M. MacDonald and Dr. S. P. Rawson for pathological and radiological investigations; to colleagues who have assisted in undertaking cardiological investigations; and to the Board of Management and to the private benefactors of the hospital for their generous provision of equipment.

\section{REFERENCES}

Bonham Carter, R. E. (1955). Quoted by Reinhold, J. D. L. (1958) in Modern Trends in Padiatrics (2nd series), p. 89. Butterworth, London.

Carleton, R. A., Abelmann, W. H., and Hancock, E. W. (1958). Familial occurrence of congenital heart disease. Report of three families and review of the literature. New Engl. J. Med., 259, 1237.

Gerbode, F., and Cooley, D. A. (1963). In Report of International Cardiovascular Society. Brit. med. J., 2, 863.

Holt, M., and Oram, S. (1960). Familial heart disease with skeletal malformations. Brit. Heart J., 22, 236.

Keith, J. D. (1956). Congestive heart failure. Review article. Pediatrics, 18, 491.

Muller, W. H., Jr., and Dammann, J. F., Jr. (1952). The treatment of certain congenital malformations of the heart by creation of pulmonic stenosis to reduce pulmonary hypertension and excessive pulmonary blood flow. Surg. Gynec. Obstet., 95, 213.

Ochsner, J. L., Cooley, D. A., McNamara, D. G., and Kline, A. (1962). Surgical treatment of cardiovascular anomalies in 300 infants younger than one year of age. J. thorac. Surg., 43, 182.

Senning, A. (1959). Surgical correction of transposition of the great vessels. Surgery, 45, 966.

Wade, G., and Wright, J. P. (1963). Spontaneous closure of ventricular septal defects. Lancet, 1, 737.

Zetterqvist, P. (1960). Multiple occurrence of atrial septal defect in a family. Acta prediat. (Uppsala), 49, 741. 